\title{
Composición, identidad y cardinalidad
}

\author{
Composition, Identity and Cardinality
}

\author{
Sebastián Briceño \\ Universidad de Santiago de Chile, Chile \\ jsbricen@gmail.com
}

\begin{abstract}
Resumen
En este artículo intento dar cuenta de la tesis según la cual composición es identidad y de las críticas que pueden formularse en contra de la misma. Según la versión débil de la tesis, composición, por el hecho de ser análoga a identidad en ciertos respectos importantes, es tan ontológicamente inocente como identidad. Según la versión fuerte de la tesis, composición es literalmente una especie de la relación genérica de identidad, y es tan ontológicamente inocente como toda especie de identidad. Como veremos, la versión débil incurre en una petición de principio o en un non sequitur; y la version fuerte es incoherente y enfrenta un dilema. Así, composición no es identidad: una cosa no puede ser idéntica a varias cosas.
\end{abstract}

Palabras clave: todo, partes, unidad, plurales, indiscernibilidad de los idénticos, inocencia ontológica, compromiso ontológico.

\begin{abstract}
In this article I attempt to give an account of the thesis according to which composition is identity and of the criticisms that might be formulated against it. According to a weak version of the thesis, composition, in virtue of being analogous in important respects to identity, is as ontologically innocent as identity. According to the strong version of the thesis, composition is literally a species of the generic relation of identity, and is as ontologically innocent as any species of identity. As we will see, the weak version begs the question or incurs in a non sequitur; and the strong version is incoherent and faces a dilemma. Thus, composition is not identity: one thing cannot be identical to many things.
\end{abstract}

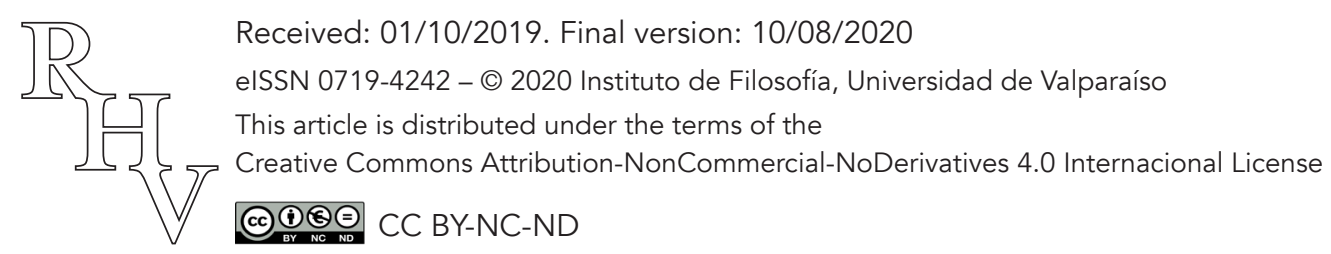


Keywords: whole, parts, unity, plurals, indiscernibility of identicals, ontological innocence, ontological commitment.

\section{Identidad, unidad y composición}

Identidad (ser el mismo objeto) y unidad (ser un objeto) son propiedades necesariamente conectadas. $\mathrm{O}$ al menos así parece ser.

Entiendo que la identidad es la relación lógica primitiva que todo objeto tiene consigo mismo y con ningún otro objeto (Frege 1960, 56). Admite ser caracterizada en términos de las siguientes dos leyes fundamentales (Wiggins 2016, 3-5):

Reflexividad: para todo $x, x=x$.

PII: para todo $x$, para todo $y$, si $x=y$, entonces, para toda propiedad F, $x$ es $\mathrm{F}$ si y solo si $y$ es F.

De estas dos leyes fundamentales es posible deducir otras leyes que hacen de la identidad la relación de equivalencia única sujeta a PII (Wiggins 2016):

Simetría: para todo $x$, para todo $y$, si $x=y$, entonces $y=x$.

Transitividad: para todo $x$, para todo $y$, para todo $z$, si $x=y$ e $y=z$, entonces $x=z$.

Determinación Absoluta: para todo $x$, para todo $y$, si $x=y$, entonces es absolutamente determinado que $x=y$.

Necesidad: para todo $x$, si $x=x$, entonces necesariamente $x=x$.

Permanencia: para todo $x$, para todo $y$, si $x=y$, entonces siempre $x=y$.

En lo que sigue, aceptaré las leyes anteriores como axiomas.

Todo objeto, además de ser idéntico a sí mismo y distinto a cualquier otro, es uno: un objeto, una unidad, un individuo, una cosa. No es varias cosas. Una cosa es necesariamente idéntica a sí misma; dos cosas son necesariamente distintas entre sí. Si " $x$ " e " $y$ " son expresiones singulares que refieren, y el enunciado " $x=y$ " es verdadero, entonces el referente de " $x$ " e " $y$ " es $u n$ objeto, el mismo objeto. Si el enunciado " $x \neq y$ " es verdadero, entonces los referentes de " $x$ " e " $y$ " son dos objetos, distintos objetos.

$\mathrm{Si}$, como afirmo, identidad y unidad son propiedades necesariamente conectadas, entonces un objeto idéntico a sí mismo no solo no puede ser distinto a sí mismo, sino que tampoco puede ser dos objetos. Para que la propiedad ser dos sea instanciada, se requieren al menos dos objetos: ser dos es una propiedad plural, una propiedad instanciada por varios individuos (Yi 1999b; McDaniel 2013). Y toda vez que hay dos objetos, hay dos objetos distintos, esto es, se instancia la relación ser distinto a. 
En el sentido expuesto se pronuncia Leibniz:

Sostengo como axiomática la proposición idéntica que varía solo en énfasis: lo que no es verdaderamente una entidad tampoco es verdaderamente una entidad. Siempre se ha pensado que 'uno' e 'identidad' son intercambiables. Entidad es una cosa, entidades otra; pero el plural presupone el singular, y donde no hay entidad, menos habrá varias entidades. (Mason 1967, 121, énfasis en el original)

Y Russell lo siguió de cerca:

Cualquier cosa que es, es uno: ser y uno, como Leibniz comenta, son términos convertibles. Es difícil estar seguro de hasta qué punto esas aserciones son meramente gramaticales. Pues, aunque cualquier cosa que es, es uno, es igualmente verdadero que cualesquiera cosas que son, son varias. $(2010,133)$

De otro lado, la relación de composición es la relación varios-uno que se da entre las partes y el todo o fusión que ellas componen. Tiene la forma las $x x$ componen $y$, donde las $x x$ son variables plurales y la $y$ una variable singular, de modo que la relación es instanciada, en un extremo, por las varias partes de un todo o fusión y, en el otro extremo, por el todo o fusión que esas varias partes componen. La relación las xx componen y es la conversa de y es la fusión de las $x x$. Un todo o fusión, cualquier todo o fusión, es un objeto, unidad, individuo o cosa. Una parte, cualquier parte, también es un objeto, unidad, individuo o cosa. Tanto el concepto todo como el concepto parte parecen suponer como condición de aplicación un dominio de cuantificación plural en el cual hay al menos un objeto compuesto por varios objetos. Es decir, para que haya todo y partes en un sentido propio no puede haber solo uno o varios átomos mereológicos. Si este último fuese el caso, entonces estaríamos frente a uno o varios objetos que solo caerían impropiamente bajo los conceptos parte o todo, pues solo lo harían en tanto cada uno de ellos está compuesto exclusivamente por sí mismo y no compone más objetos que sí mismo, esto es, solo en tanto cada uno de ellos es idéntico a sí mismo. Así, por ejemplo, el objeto eleático no es ni un todo ni una parte en sentido propio, pues carece de partes y no es parte de nada. Solo cae bajo los conceptos parte o todo en sentido impropio, un sentido que no presupone complejidad mereológica de ningún tipo. En contraste, la cabeza de Sócrates no solo es una parte de Sócrates, sino que además es un todo compuesto de varias partes: orejas, ojos, nariz, etc. Aquí los conceptos todo y parte se aplican en un sentido propio. Como se puede apreciar, utilizo las expresiones "parte propia" y "parte impropia" en el sentido técnico de la mereología estándar: $x$ es parte propia de $y$ si y solo si $x$ es parte de $y$ y $x$ no es idéntico a $y$; en contraste, $x$ es parte impropia de $y$ si y solo si $x$ es idéntico a $y$ (Simons 1987, 9-16). Por analogía, entiendo que un todo en sentido propio es un todo compuesto de partes propias, y un todo en sentido impropio es un todo compuesto solo por una parte impropia: sí mismo. En lo que sigue, cuando hablo de todos y de partes estoy hablando de todos y de partes en sentido propio. 
Que varios objetos compongan un objeto no es lo mismo (no es idéntico a) que varios objetos sean idénticos a un objeto. Necesariamente, todo objeto es un objeto, varios objetos son más de uno, y varios objetos no son un objeto. En un slogan: Composición no es Identidad. Esta es la tesis que subyace a este artículo. Mi objetivo es defenderla por la vía negativa. Quiero atacar la crecientemente popular tesis Composición como Identidad, según la cual, en términos generales, el todo no sería "nada por sobre o por encima" de las partes, por lo que composición sería, en algún sentido a determinar, identidad. Si esto fuese cierto, entonces varias cosas podrían ser (idénticas a) una cosa, contra la tesis que defiendo.

Ninguna de las ideas discutidas aquí es nueva. El problema, desde luego, nos acompaña al menos desde Parménides; la tesis que apoyo encuentra antecedentes en Leibniz y Russell, a quienes cité al comienzo; la tesis que ataco encuentra antecedentes en Platón y Frege, a quienes citaré más adelante; y ambas tesis cuentan con defensores contemporáneos. Así, a favor de alguna versión de Composición como Identidad encontramos a Armstrong (1997), Baxter (1988; 2014), Bohn (2014), Bricker (2016), Lewis (1991), y Wallace (2011a; 2011b); y en contra de la misma encontramos a Koslicki (2008), McDaniel (2008), Merricks (1999), Sider (2013, 2014), van Inwagen (1994; 2002), y Yi (1999a; 2014). Mi objetivo, por tanto, más que defender una tesis original, es arrojar luces sobre el estado de la cuestión, identificar y evaluar los principales argumentos en juego, y dejar de manifiesto la implausibilidad de la tesis que ataco, no obstante la creciente adhesión que parece despertar.

Un examen de la literatura relevante indica que Composición como Identidad puede adoptar tres formas distintas: una débil (CID), una fuerte (CIF), y una extraña (CIE). Conviene distinguirlas y tratarlas por separado, pues no dicen lo mismo ni tienen las mismas implicancias. Así lo haré en lo que sigue. Por razones de espacio, aquí solo me ocuparé de CID y de CIF.

CIE, la tesis defendida por Baxter $(1988 ; 2014)$, deberá quedar para otra ocasión. Solo anticiparé que, como CIE supone abrazar una forma de relativismo ontológico radical, podemos decir en su contra algo parecido a lo que muchos dicen en contra de quienes, siguiendo a Geach (1967), defienden la idea de identidad relativa: se trata de una propuesta tan revisionista de nuestra comprensión tradicional de la identidad que, más que ofrecer un nuevo tratamiento de un viejo tema, lo que hace es cambiar de tema. Digo que CIE supone abrazar una forma de relativismo ontológico radical porque, según ella, por un lado, identidad, número y existencia serían cuestiones relativas a lo que Baxter llama "conteos" ("counts"), y, por otro lado, contra PII, los idénticos son discernibles, de modo que una cosa puede cualitativamente diferir de sí misma. Dentro de un mismo conteo, nunca varias cosas son idénticas a una cosa. Pero a través de distintos conteos ("inter-conteo", “cross-count"), varias cosas sí pueden ser idénticas a una cosa. Hay un conteo para el todo y otro conteo para las partes. Si contamos el todo, no contamos las partes; si contamos las partes, no contamos el todo. El todo es literalmente idéntico "inter-conteo" a las partes. 
Así, por ejemplo, un six-pack de latas de cerveza es una cosa relativa a un "conteo" y seis cosas relativas a otro "conteo". So pena de contradicción, una lata de cerveza no es idéntica a seis latas de cerveza, y un six-pack no es idéntico a seis six-packs. Pero seis latas de cerveza son literalmente idénticas "inter-conteo" a un six-pack. La relatividad que propone CIE no es conceptual, pues qué es idéntico/distinto a qué no es algo que dependa de medios de concebir o representar, sino de las cosas en sí. De ahí proviene buena parte de su radicalidad y, como veremos, su distancia con CIF. Adicionalmente, CIE entiende que un idéntico puede ser discernible de sí mismo si se está dispuesto a abrazar una ontología de aspectos para tratar la relación entre las partes y el todo. Los aspectos son típicamente capturados mediante expresiones tales como "en tanto que" o "qua". Así, por ejemplo, podemos afirmar, aparentemente sin contradicción, que una misma cosa, Juan, es estricto qua profesor y no es estricto qua padre. Estos aspectos de Juan no serían varias cosas distintas de las cuales Juan está compuesto. Se trataría, más bien, de diversas formas de discernir a Juan de sí mismo (Baxter 1988, 200-201; 2014, 252; Turner 2014, 231-232).

\section{CID}

Partamos entonces por CID. Según CID, la relación de composición es como la relación de identidad. A veces se le atribuye esta tesis a Lewis, apelando a pasajes como el siguiente:

Digo que composición - la relación de parte a todo, o, mejor, la relación varios-uno de varias partes a su fusión - es como identidad. El 'son' de composición es, por decirlo así, la forma plural del 'es' de identidad. Llamemos a esto la Tesis de Composición como Identidad. Es en virtud de esta tesis que la mereología es ontológicamente inocente: nos compromete solo a cosas que son idénticas, por decirlo así, a lo que estábamos comprometidos desde antes. (1991, 82, énfasis agregado)

La tesis no afirma simplemente que entre composición e identidad hay semejanza o analogía. Esto es trivial. En algún sentido, cualquier cosa es como cualquier otra cosa. Desde ya, por ejemplo, cualesquiera dos cosas son cosas, cada una de ellas idéntica a sí misma y una en número. El punto central de la tesis no es tanto la defensa de una semejanza entre dos relaciones distintas, sino la defensa de una semejanza en grado tal que la composición sería tan "ontológicamente inocente" como la identidad. En este sentido, composición sería, "por decirlo así, identidad. Es la semejanza entre composición e identidad el hecho que fundaría la supuesta inocencia ontológica de la composición mereológica. Y es la supuesta inocencia ontológica de la composición mereológica el punto central de la tesis de Lewis: 
La mereología es ontológicamente inocente.

Para estar seguros, si aceptamos la mereología, estamos comprometidos con la existencia de todo tipo de fusiones mereológicas. Pero dado un compromiso previo con gatos, digamos, un compromiso con fusiones-de-gatos no es un compromiso adicional. La fusión no es nada por sobre y encima de los gatos que la componen. Ella simplemente es ellos. Ellos son ella. Tómalos juntos o tómalos por separado, los gatos son la misma porción de Realidad de cualquier forma. [...] Si haces un inventario de la Realidad de acuerdo a tu esquema de las cosas, sería contar dos veces listar los gatos y luego listar su fusión. En general, si ya estás comprometido con la existencia de algunas cosas, no incurres en ningún compromiso adicional cuando afirmas su fusión. (1991, 81-82, énfasis agregado)

Hay varias cuestiones dignas de atención en los pasajes citados.

Partamos por la idea de compromiso (ontológico). Digamos, por ejemplo, que yo soy un monista eleático: creo que solo existe una cosa, UNO, y que, por tanto, todo es idéntico a UNO. Digamos que tú eres más sensato y que, ante mi osada propuesta, intentas rebatirme apelando a lo que se presenta como obvio frente a nuestras narices. Podrías, sin ir más lejos, decirme que todo parece indicar que ahora estamos teniendo un diálogo filosófico y que eso requiere que existan al menos dos cosas, presupuesto que obviamente parece cumplirse: tú existes, yo existo, tú y yo somos distintos; por tanto, hay más de una cosa. Yo, para salvar mi extraña cosmovisión, me vería forzado a decirte que, en ese mundano sentido en el que tú pareces entender la existencia, claramente hay más de una cosa, pero tu problema es que confundes apariencias y realidad: tú y yo, aunque somos aparentemente dos cosas distintas, somos realmente una y la misma cosa, UNO. Lo que a ti se te presenta como un diálogo es realmente un monólogo. Cuando digo que existe solo una cosa quiero decir que existe realmente solo una cosa, aunque las apariencias digan lo contrario. La posición del monista eleático es extraña. Todo parece indicar que es falsa. Pero, hecha la aclaración anterior, no es una posición inconsistente; por tanto, posiblemente es verdadera. Es decir, bien podemos hacer sentido de ella si distinguimos, como hice implícitamente, entre dos formas de compromiso ontológico, una cualificada y otra no cualificada. El compromiso ontológico del monista eleático es cualificado: su teoría no se refiere a lo que existe simpliciter, sino a lo que existe realmente, por oposición a lo que existe solo aparentemente. Si cuando él dice "existe solo una cosa, UNO" lo interpretamos como usando el cuantificador existencial en forma absolutamente irrestricta, entonces se auto-refutaría en el acto de verbalizar su teoría, pues la sola palabra "UNO" está compuesta de tres objetos distintos: "U", "N", y "O". Pero una interpretación caritativa exige tomarlo como usando el cuantificador existencial en términos implícitamente más restringidos: como refiriéndose solo a lo nombrado por su teoría, no a los medios de representación desplegados por ella. El punto es generalizable: para poder decir algo sobre algo, no podemos nombrar al mismo tiempo todo medio de representación. En general, 
para decir algo con sentido, para evitar un discurso totalmente impredicativo, debemos renunciar a hablar en términos absolutamente globales o irrestrictos (Bohn 2014, 147; Priest 2011). En algún punto tendremos que parar de cuantificar sobre medios de representación para poder decir algo sobre lo representado por ellos. Una forma de evitar estas consecuencias es precisamente introduciendo, explícita o implícitamente, restricciones al cuantificador existencial. (Esta es una buena razón para entender, siguiendo a Fine (2009), que la pregunta central de la ontología no es, como sostenía Quine, qué existe, sino qué existe realmente. Y podemos apelar, como lo hace el mismo Fine, a un concepto primitivo de realidad, pues es dudoso que podamos definirlo en términos no circulares.)

El monismo eleático, entonces, tal como lo he caracterizado aquí, equivale a lo que Schaffer (2010a; 2010b) llama "monismo existencial", la posición según la cual, en el dominio de los objetos concretos, realmente existe solo un objeto, un solo simple extenso. Lo que luego identificamos como partes de ese todo no serían más que partes bajo una "ficción de descomposición" de dicho todo (Schaffer 2007; Rosen y Dorr 2002). A estas ficciones yo he preferido llamarlas aquí "apariencias", aunque tanto el concepto ficción como el concepto apariencia pueden funcionar, alternativamente, como par dicotómico del concepto realidad. El monismo eleático o existencial es distinto a lo que Schaffer llama "monismo prioritario". Según este último, tanto el todo como las partes realmente existen (las partes no son apariencias oficciones), pero conviven en una relación de prioridad ontológica conforme a la cual el todo es prioritario a sus partes; es decir, el dominio de los objetos concretos tiene cardinalidad mayor a $1 \mathrm{y}$ es metafísicamente estructurado. Como se ve, el monismo eleático o existencial tiene compromisos ontológicos distintos al monismo prioritario. Un monismo más radical que el monismo eleático aquí articulado, un monismo según el cual solo una cosa existe simpliciter, es seguramente inefable y tal vez contradictorio (Della Rocca 2012).

Pues bien, lo anterior vale también para el debate que aquí me ocupa. Si entendemos que la posición del monista eleático se refiere a lo realmente existente, entonces se trata de una posición consistente (de hecho, que realmente no exista nada, ni siquiera UNO, sino solo apariencias, también es una posición consistente). Y lo mismo vale para Lewis. Él entiende que la realidad sí está estructurada mereológicamente. Para Lewis, realmente existen cosas que instancian la relación de composición. No cree que existan solo apariencias de todos y partes. No cree que solo existan realmente gatos y que la fusión de todos los gatos sea solo apariencia, algo que, aunque existe simpliciter (e.g., como ficción, proyección subjetiva, objeto puramente intencional, etc.) no existe realmente. Para él, existen realmente tanto gatos como fusiones de gatos. Y mucho más que gatos y fusiones de gatos, por cierto. Es el nihilista composicional quien sostiene que realmente no hay objetos compuestos, y que toda complejidad mereológica está relegada al ámbito de las apariencias. Pero Lewis no es un nihilista composicional. 
Como sea, lo relevante aquí no es si acaso las teorías del monista, del nihilista, o del mereólogo son verdaderas o falsas. El punto es a qué nos comprometen en caso de ser verdaderas. Y, como se puede apreciar, nos comprometen a cosas distintas.

Vamos ahora a la idea de inocencia ontológica. Asumiendo que hablamos de lo realmente existente, podemos decir que la posición del monista eleático es ontológicamente inocente, al menos en el siguiente sentido. Aunque use una frase que contiene más de una palabra para describir su posición, el compromiso ontológico del monista eleático es solo con la existencia de una cosa: UNO. El monista eleático no incurre en adición ontológica cuando dice que hay algo que tú y yo somos (idénticos a): UNO. La referencia de "tú", "yo", "todo", "eso" es uno y el mismo objeto. Cualquier cosa es idéntica a UNO. No hay nada que sea distinto a UNO. Si al monista eleático su contendor le reprocha que cuando él dice "tú y yo somos idénticos" ya está reconociendo, en algún leve sentido, al menos como objetos puramente intencionales, la existencia de dos objetos distintos, entonces él puede replicar diciendo que está hablando de lo realmente existente, no de lo aparentemente existente. Es decir, el monista eleático puede aceptar la relación de identidad y toda su lógica sin adquirir compromisos ontológicos adicionales a la existencia de UNO. Nótese que el monista eleático también puede aceptar la mereología como una teoría verdadera sin incurrir en mayores compromisos ontológicos a los que ya ha asumido. Como UNO no es parte de otras cosas ni está compuesto de otras cosas distintas a sí mismo, UNO solo es un todo y una parte en sentidos impropios. Sucederá que toda la referencia de expresiones como "todo" y "partes" será agotada por uno y el mismo objeto. Y mientras la expresión singular "todo" solo aparentemente nombrará un objeto compuesto, la expresión plural "partes" será solo mala gramática para nombrar lo irreductiblemente singular.

¿Pero puede decir lo mismo Lewis, quien claramente es un pluralista y un creyente en la mereología, alguien que acepta la real existencia de más de una cosa compuesta? Para responder, simplifiquemos la realidad. Digamos que tú existes, que yo existo, y que tú y yo somos distintos. Hasta aquí nuestra teoría nos compromete con un pluralismo mínimo: realmente hay dos cosas. A la lógica propia de singulares, se suma entonces la lógica propia de plurales, con sus términos o variables plurales (e.g., "ellos", “ $x x$ ”, etc.), cuantificadores plurales (e.g., "todas las cosas son...", "hay algunas cosas que..."), y un predicado plural especial cuyo segundo argumento admite términos plurales y singulares: “...es uno de..." (Boolos 1984; Lewis 1991, 62-71; Yi 2005; 2006). ¿Qué pasa si complementamos nuestra teoría pluralista con la mereología? Según la formulación estándar de la mereología que hace Lewis $(1991,72-74)$, si tomamos ser parte de como primitivo, podemos proceder a hacer otras definiciones básicas. En primer lugar, podemos decir que dos cosas se traslapan si y solo si tienen una parte en común, y son distintas si y solo si no tienen una parte en común. También podemos decir que una cosa es una fusión de varias 
cosas si y solo si la primera tiene a las segundas como partes y no tiene otra parte que sea distinta a alguna de las segundas. Lewis prosigue incorporando tres axiomas mereológicos de general aceptación:

Transitividad: si $x$ es parte de $y$, e $y$ es parte de $z$, entonces $x$ es parte de $z$.

Composición Irrestricta: siempre que hay varias cosas, $x x$, existe la fusión de estas cosas, $y$.

Unicidad de Composición: siempre que hay varias cosas, $x x$, ellas componen una única fusión, $y$; es decir, nunca hay dos fusiones con las mismas partes.

Según esta mereología básica, dado que, por ejemplo, Sócrates y Platón existen, entonces existe algo que es la fusión de Sócrates y Platón, a saber: SoPa. Sócrates y Platón son distintos: Sócrates no es parte de Platón; Platón no es parte de Sócrates. Sócrates es uno de Sócrates y Platón; Platón es uno de Sócrates y Platón. Pero hay algo, un objeto, distinto a Sócrates y Platón, del cual ambos son partes, SoPa. Si Sócrates afirma su propia existencia y luego afirma la existencia de un objeto idéntico a sí mismo, entonces no incurre en un compromiso ontológico adicional. Si luego afirma la existencia de Platón, y Platón es distinto a Sócrates, entonces Sócrates sí incurre en un compromiso ontológico adicional, pues ahora hay dos cosas: Sócrates y Platón. Si luego Sócrates afirma que cree en una teoría según la cual hay algo, una fusión, que existe automáticamente cada vez que varias cosas existen-es decir, si Sócrates, siguiendo cándidamente a Lewis, cree en una teoría que contiene el axioma de Composición Irrestricta-parece obvio que, si es que está comprometido inicialmente con su propia existencia y con la existencia de Platón, entonces automáticamente se ha comprometido con la existencia de una tercera cosa, la fusión de la cual él y Platón son partes: SoPa.

¿Cómo es que Lewis elude este compromiso ontológico adicional? Diciendo que composición, al ser como identidad, sería tan ontológicamente inocente como ella. Así, SoPa no sería adición ontológica a la existencia de Sócrates y a la existencia de Platón, en similar sentido a como un objeto idéntico a Sócrates no es adición ontológica a Sócrates. Si hiciéramos un inventario de la Realidad incluyendo a Sócrates y a Platón, sería redundante incluir además a SoPa, y vice-versa, pues "son la misma porción de Realidad". Sócrates y Platón componen SoPa, y composición es como identidad: Sócrates y Platón son, por decirlo así, idénticos a SoPa. Lewis entiende que la analogía entre composición e identidad da "el significado" de la tesis Composición como Identidad (Lewis 1991, 87), y que dicha analogía se basa en cinco aspectos (Lewis 1991, 85-86).

A continuación, siguiendo de cerca a Yi (1999a), paso a exponer y criticar cada uno de los aspectos en los que se basa la analogía: 
En primer lugar, se supone que así como Sócrates no tiene que satisfacer ninguna condición especial para que exista algo idéntico a sí mismo, las $x x$ tampoco tienen que satisfacer ninguna condición especial para que exista algo que ellas componen, $y$ [por Composición Irrestricta].

Réplica: Pero si yo creo en una teoría según la cual, dada la existencia de una cosa, existe automáticamente otra cosa, entonces, si la teoría es verdadera, esta segunda cosa no tiene que satisfacer ninguna condición especial para existir, como tampoco tiene que satisfacer ninguna condición especial la tercera cosa que automáticamente existe una vez que existe la segunda, y así, ad infinitum. Por ejemplo, si yo creo en teoría de conjuntos, y la teoría de conjuntos es verdadera, entonces, a partir de comprometerme con nada, $\varnothing$, me comprometo automáticamente con todo el paraíso de Cantor, sin que ningún conjunto tenga que satisfacer "alguna condición especial" para existir aparte de ser idéntico a sí mismo. O si yo creo en una teoría según la cual, dada la existencia de Dios, éste, por su propia naturaleza, no puede evitar crear al Diablo, entonces, si Dios existe y mi teoría es verdadera, el Diablo no tiene que cumplir ninguna condición especial para existir aparte de ser idéntico a sí mismo. Nótese, como ya dije anteriormente, que el punto no es si acaso estas u otras teorías son falsas o verdaderas, sino si acaso son o no ontológicamente inocentes en caso de ser verdaderas.

En segundo lugar, así como no pueden existir dos cosas distintas que sean idénticas a Sócrates, no pueden existir dos fusiones distintas, $y^{\prime}$ e $y$ ”, compuestas por las mismas partes, $x x$ [por Unicidad de Composición].

Réplica: Pero el punto no es si acaso pueden existir dos fusiones, $y^{\prime}$ e $y$ ”, compuestas por las mismas partes, $x x$. El punto es que, aun cuando ese sea el caso, de ahí no se sigue que $y$ no sea adición ontológica a las $x x$. Pues, por ejemplo, también es cierto que no pueden existir dos conjuntos con los mismos miembros. Es decir, si el conjunto A tiene como miembros a Platón y Sócrates, y el conjunto B tiene como miembros a Platón y Sócrates, entonces A es idéntico a B. Pero claramente un conjunto no es (idéntico a) sus miembros: \{Platón, Sócrates\} no es idéntico a Platón y Sócrates (Fine 1994). Desde ya, el primero es una cosa, en particular un conjunto, mientras que los segundos son dos cosas, en particular dos seres humanos. Y es que un criterio de identidad de una cosa y la cosa en cuestión, no son una y la misma cosa. Lo cierto es que la identidad es, con toda seguridad, una propiedad primitiva o básica, indefinible en términos de otras cuestiones más fundamentales, pues toda definición, real o nominal, parece presuponerla; difícilmente puede concebirse algo más fundamental que el hecho que para todo $x, x=x$ (McGinn 2000). Volveré sobre esto más adelante.

En tercer lugar, está lo que Lewis llama "facilidad para describir fusiones" (1991, 85). Se supone que así como si describo a Sócrates completamente, describo completamente aquella cosa que es idéntica a él, si describo a Sócrates y a Platón completamente, enton- 
ces describo completamente la fusión SoPa. Esto valdría también para una descripción relacional: si doy la posición espacio-temporal de Sócrates y Platón, entonces doy la posición espacio-temporal de la fusión SoPa.

Réplica: Pero cuando yo describo completamente una de las dos esferas del famoso experimento mental de Black (1952), también describo completamente la otra esfera. Y sin embargo siguen siendo objetos numéricamente distintos.

En cuarto lugar, está lo que podríamos llamar "coincidencia absoluta de locación". Si una cosa es idéntica a Sócrates, entonces dicha cosa está en el mismo mundo, tiempo y lugar en que Sócrates está. De la misma manera, Lewis entiende que la fusión de SoPa está en el mismo mundo, tiempo y lugar en que están Sócrates y Platón. Una fusión tendría locación múltiple: estaría completamente localizada donde están localizadas sus distintas partes.

Réplica: En primer lugar, coincidencia espacial, temporal y de mundo de dos o más objetos no es identidad y, como no es identidad, conlleva, en principio, compromisos ontológicos adicionales a la existencia de una sola cosa. Desde luego, es perfectamente posible desde un punto de vista lógico que un objeto esté en el mismo lugar, tiempo y mundo que otro distinto. La idea que de la coincidencia entre la locación de un todo y la locación de todas sus partes sea posible inferir que composición es tan ontológicamente inocente como identidad, parece motivada por el mismo tipo de ansiedad por parsimonia ontológica que lleva a algunos a confundir constitución material con identidad, esto es, a entender que un objeto es idéntico al trozo de materia que lo constituye, e. g., que una estatua es idéntica al trozo de bronce que la constituye. Así, perdurantistas como Lewis (1986) y exdurantistas como Sider (2001) suelen entender que constitución material es identidad, precisamente basados en razones de coincidencia de locación. Pero si seguimos Reflexividad, PII y Necesidad, tal como lo he propuesto, una estatua no es idéntica al trozo de bronce que la constituye, aun cuando ambos puedan coincidir en toda locación espacio-temporal que ocupan. Pues la estatua tiene propiedades modales que el trozo de bronce no posee, por ejemplo, el trozo de bronce podría ser aplastado y seguir siendo lo que es, pero la estatua no. Por tanto, por PII, la estatua no es idéntica al trozo de bronce que la constituye. Y no se crea que el diagnóstico cambia cuando hablamos de coincidencia ya no solo temporal y espacial, sino a través de mundos posibles. Como lo ha hecho ver persuasivamente Fine (1994), aunque Sócrates y \{Sócrates\} habiten los mismos mundos posibles, lo cierto es que Sócrates no es idéntico a \{Sócrates\}. Por tanto, \{Sócrates\} constituye adición ontológica sobre Sócrates.

En segundo lugar, se supone que el principal motivo que Lewis esgrime para entender que el todo está donde están sus partes es evitar lo que de otra manera sería una misteriosa inseparabilidad de partes disyuntas, una misteriosa conexión necesaria entre distintos existentes. Así, la presunta inseparabilidad de Sócrates y Platón se explicaría porque ambos son partes de un todo, SoPa (Lewis 1991, 85-86). Pero si SoPa es capaz de remover el 
misterio de una aparente inseparabilidad es porque realmente existe y es distinto a Sócrates y Platón. El punto ha sido explotado por Schaffer (2010b) para defender la prioridad ontológica del todo sobre las partes. La idea de Schaffer es la siguiente: en principio, toda entidad fundamental es modalmente libre; es decir, fallas en la libre recombinación de dos cosas distintas y fundamentales demandan una explicación. Y hay dos buenas explicaciones a la mano: que ambas cosas se traslapan o que ambas cosas son partes propias de un todo. Si estamos frente a cosas disyuntas, tales como Sócrates y Platón, entonces su falta de libre recombinación solo se puede explicar porque, contra lo que suponíamos, ellas no son fundamentales, sino que son partes propias dependientes de un tercer objeto que sí es fundamental y que sí exhibe libertad modal. Pero esta explicación exige que este todo no sea idéntico a sus partes, que composición no sea identidad. En efecto, es contenido fundamental de la tesis de la prioridad ontológica del todo sobre las partes defendida por Schaffer $(2010 \mathrm{a} ; 2010 \mathrm{~b})$ que tanto el todo como las partes existan y que sean distintos, pues solo entonces el todo puede ser ontológicamente prioritario con respecto a las partes.

El quinto aspecto al que Lewis apela es precisamente la supuesta inocencia ontológica de la mereología.

Réplica: Es aquí donde quienes nos oponemos a Composición como Identidad trazamos el límite. Composición mereológica e identidad podrán tener muchos parecidos, pero lo que las distingue es justamente que identidad es ontológicamente inocente y composición mereológica no. Si esto es así, entonces la supuesta inocencia ontológica de la composición mereológica no puede figurar como un aspecto fundante más de la analogía con identidad sin incurrir en una petición de principio: sería incluir dentro de las premisas del argumento la conclusión a la que el argumento pretende llegar. Lewis mismo entiende que es la analogía entre composición mereológica e identidad lo que funda la supuesta inocencia ontológica de la primera. Si esto es así, entonces esta inocencia ontológica no puede figurar como elemento integrante de la misma analogía que supuestamente le sirve de fundamento. Por esta misma razón es que Bricker (2016) yerra al quejarse de que Yi (1999a), en su ataque a CID, no considere este quinto aspecto de la analogía.

He criticado separadamente cada uno de los aspectos fundantes de la analogía. Pero tal vez colectivamente son capaces de hacer lo que individualmente no pueden. Pues bien, si para evitar la petición de principio descartamos el quinto aspecto y no presuponemos, en consecuencia, la inocencia ontológica de la composición mereológica, que es aquello que se requiere probar, ¿se sigue de los demás aspectos integrantes de la analogía tomados conjuntamente que, dada la existencia de Platón y dada la existencia de Sócrates, entonces la existencia de la fusión SoPa no es un compromiso ontológico adicional? Claramente no. Se trata de un argumento evidentemente inválido. Según CID, composición no es identidad, sino algo parecido a la identidad, algo como la identidad. Pero si la relación de composición es solo "parecida a", "análoga a", o "como" la relación de identidad, entonces la inocencia ontológica de la mereología no se sigue como conclusión. Estamos frente a un trivial non sequitur (Yi 1999a, 153). Y es que, trivialmente, la analogía o similaridad 
entre $x$ e $y$ es insuficiente para garantizar la identidad entre $x$ e $y$. Sería como inferir que tú, que eres distinto a mí, no representas adición ontológica a mi existencia a partir del hecho que tú y yo nos parecemos mucho. Si yo creo en una teoría de clases según la cual, dada la existencia de manzanas, existe entonces la clase de las manzanas, tengo una teoría que, de ser verdadera, satisface los cuatro aspectos en los que Lewis funda su analogía. Y, sin embargo, la clase de las manzanas es adición ontológica a las manzanas. Desde luego, ella tiene propiedades que las manzanas no tienen: ella es una, mientras que las manzanas son varias; una manzana pertenece a la clase de las manzanas; la clase de las manzanas no; etc. Por tanto, por PII, son cosas distintas. Lo mismo sucede si creo en una teoría de duplicados coincidentes como la que propone Yi (1999a, 150). Si esta teoría sostiene que, dada la existencia de un objeto, existe un duplicado exacto suyo que comparte no solo sus propiedades intrínsecas sino también su locación temporal, espacial y de mundo, entonces por cada objeto hay otro objeto adicional. Si esta teoría es verdadera, entonces tu existencia trae consigo la existencia de un duplicado exacto tuyo. Seguramente es una teoría falsa, pero el punto es que no es inconsistente y que no es ontológicamente inocente.

El problema parece estar en la frase "son la misma porción de Realidad". Como hemos visto, la mereología no es ontológicamente inocente. Si ella es verdadera, entonces tanto el todo como las partes que "son la misma porción de Realidad" han de incluirse en el inventario del mundo. Para evitar el compromiso ontológico que conlleva esta última posición, la frase se interpreta por Lewis y sus seguidores como si el todo y las partes compitieran por el mismo espacio, como si estuvieran gobernados por una suerte de principio de impenetrabilidad tal que su coincidencia se torna imposible. Si el todo y las partes coinciden, entonces es porque el todo es, por decirlo así, idéntico a las partes. Coincidencia solo es tolerable si colapsa en identidad. Contar objetos coincidentes sería contar dos veces lo mismo. Pero tal principio de impenetrabilidad es sospechoso desde un punto de vista lógico, pues su negación no es una contradicción; y también es sospechoso desde un punto de vista empírico, pues, si hemos de creer a algunos físicos, objetos tales como campos o partículas sub-atómicas parecen no estar gobernados por tal restricción. Irónicamente, quienes defienden alguna variante de Composición como Identidad, suelen alegar que sus contradictores tienen un concepto de objeto metafísicamente demandante o sustancioso en mente, y que es esto lo que les impediría ver el carácter inocente de su propuesta, que solo descansaría sobre un concepto lógico o metafísicamente leve de objeto (Bohn 2014; Bricker 2016). Por el contrario, justamente porque mi concepto de objeto no es más sustancioso ni demandante que el de objeto lógico, es que no veo contradicción alguna en la existencia de objetos coincidentes. Son los que defienden alguna variante de Composición como Identidad quienes, por alguna extraña razón, atribuyen a los objetos una supuesta impenetrabilidad que les impediría coincidir con objetos distintos, una propiedad que en principio parece totalmente ajena a objetos lógicos o metafísicamente leves. 


\section{CIF}

Si CID no asegura la inocencia ontológica de la composición, y, por tanto, que composición sea como identidad en ese sentido mínimo, ¿por qué no simplemente sostener que composición es literalmente identidad entre el todo y las partes? Esto es justamente lo que hace CIF. Una solución semejante parece poner Platón en boca de Sócrates. En Teeteto, por ejemplo, Sócrates afirma lo siguiente: "[p]orque en algo que tiene partes, es necesario que el todo sea la suma de las partes" (Platón 1998, 303). Y una idea similar desarrolla en Parménides:

[S]i alguien demostrara que yo soy uno y múltiple, ¿por qué habría de sorprendernos?; bien podría decir, cuando pretendiese mostrar que soy múltiple, que unas son las partes derechas de mi cuerpo y otras las izquierdas, unas las anteriores y otras las posteriores, e, igualmente, unas las superiores y otras las inferiores (yo creo, por cierto, que participo de la multiplicidad); y cuando pretendiese mostrar que soy uno, podría decir que, del grupo de nosotros siete, yo soy un único hombre, porque participo también de lo uno. De ese modo, ambas afirmaciones se muestran verdaderas. (Platón 1998, 40)

CIF también es rastreable en algunos pasajes de Lewis:

[Las relaciones mereológicas] son sorprendentemente análogas a la identidad ordinaria, la relación uno-uno que cada cosa tiene consigo misma y con ninguna otra. Tan sorprendente es esta analogía que resulta apropiado marcarla por la vía de hablar de las relaciones mereológicas-la relación varios-uno de composición, las relaciones uno-uno de parte a todo y de traslape-como tipos de identidad.

Identidad ordinaria es el caso especial, límite, de identidad en el sentido amplio. (1991, 84-85, énfasis agregados)

El problema de CIF es que es incoherente. La incoherencia se produce justamente cuando se intenta llevar la analogía o semejanza entre composición e identidad al límite que el propio Lewis admite como infranqueable. Sucede que los idénticos son indiscernibles; en cambio, el todo y las partes son discernibles. El todo y sus partes, desde ya, tienen propiedades numéricas distintas: el todo es una cosa; sus partes son varias cosas. Lo que es verdad del todo no siempre es verdad de las partes. Luego, por PII, el todo no es, no puede ser, idéntico a las partes. Ahora, la contradicción no puede ser imputada a Sócrates en Teeteto si es que por "suma" él entiende un objeto numéricamente distinto a las partes, pues entonces Sócrates no estaría diciendo algo diferente a que el todo es el todo. La contradicción solo le es imputable si el término "suma" es entendido como un término plural que refiere a las varias partes de un todo. 
CIF debe entonces introducir algunas restricciones que hagan aceptable lo que a primera vista es incoherente. Estas restricciones buscan, por un lado, explicar la supuesta inocencia ontológica de la composición mereológica y, por otro lado, que esta supuesta inocencia ontológica no conlleve poner en entredicho PII. Se trata de las siguientes: (i) el todo ha de ser entendido como idéntico a sus partes tomadas colectivamente, y no distributivativamente, y (ii) la relación de identidad ha de ser entendida como una relación genérica.

Según CIF, la fórmula $A=B$ no es una fórmula básica donde $A$ y $B$ son variables singulares, como usualmente suponemos, sino una fórmula genérica, donde $A$ y $B$ son variables que admiten valores de cualquier cardinalidad. Así, si tanto $A$ como $B$ adoptan valores de cardinalidad singular, tenemos una instancia de la familiar relación de la lógica singular: $x=y$. En seguida, si tanto $A$ como $B$ adoptan valores de cardinalidad plural, entonces tenemos una instancia de la relación de la lógica de plurales: $x x=y y$. Finalmente, si $A$ adopta valores de cardinalidad plural y $B$ adopta valores de cardinalidad singular, tenemos el caso híbrido de identidad: $x x=y$. Es en esta instancia híbrida donde CIF sostiene que el que las $x x$ compongan $y$ es (idéntico a) que las $x x$ sean (idénticas a) $y$. Así, CIF admite ser entendida en términos definicionales, tal como lo hace Bohn $(2014,144)$ :

$$
x x \mathrm{C} y={ }_{\mathrm{df}} x x=\mathrm{y}
$$

Pero CIF es incorrecta. En primer lugar, entender la relación de identidad como una relación genérica es una propuesta artificiosa y ad-hoc, pues introduce deliberadamente una ambigüedad donde no la hay solo con el fin de acomodar la idea que la relación de composición sea tratada como una especie de identidad. Creo que McGinn (2000) ha demostrado con solvencia que la identidad es una propiedad lógica unitaria, indefinible, fundamental, y genuina. Resulta más o menos evidente que la identidad es una propiedad que no puede ser definida en términos de otras; que muchas otras propiedades reposan sobre ella; y que, por mucho que todo objeto la tenga, no por ello deja de ser real o genuina. Asimismo, es difícil imaginar que uno pueda siquiera comprender el contenido proposicional más elemental sin presuponer identidad singular. Desde luego, es claro que solo comprendemos la lógica de plurales porque comprendemos la lógica de singulares; y solo podemos llegar a comprender relaciones como composición, constitución, predicación, etc., o principios tales como el del tercio excluido o el de no contradicción, porque comprendemos la identidad singular (McGinn 2000). Pero no viene al caso detenerme en todos estos rasgos. Sí quiero detenerme en el carácter unitario de la identidad, que es lo que aquí interesa. Creo, como McGinn, que Frege captura perfectamente el carácter unitario de la identidad cuando sostiene que esta "es una relación que se nos presenta de tan específica manera que es inconcebible que varias formas de ella puedan ocurrir" (McGinn 2000, 1). La identidad no admite tipos, variaciones, cualificaciones. La identidad no es un género con varias especies o una propiedad determinable; la identidad no es relativa; la identidad no admite variedades conforme a distintos "criterios de identidad". Si a veces 
nos sentimos tentados a tratar la identidad en forma no unitaria es porque la identidad relaciona distintos tipos de objetos. Pero que existan varios tipos de relata no es lo mismo a que existan varios tipos de relaciones. Identidad siempre es la misma relación, con sus mismas propiedades y consecuencias lógicas, aunque haya distintos tipos de objetos idénticos a sí mismos. Así, del hecho que la identidad rija entre objetos abstractos y también entre objetos concretos no se sigue que haya algo así como dos tipos de identidad, abstracta y concreta. $\mathrm{O}$ del hecho que la identidad gobierne propiedades determinables no se sigue que la relación de identidad sea determinable. Ser rojo es determinadamente idéntico a ser rojo, aunque ser rojo sea una propiedad determinable con respecto a la propiedad ser escarlata. Y cuando se distingue, por ejemplo, entre identidad cualitativa e identidad numérica, no se distingue, en realidad, entre dos tipos de identidad, sino entre dos tipos de relata, a saber: objetos particulares y objetos universales. La identidad es siempre identidad numérica. Decir que $x$ e $y$ son cualitativamente idénticos, pero numéricamente distintos, no es más que una forma confusa de decir que propiedades numéricamente idénticas son instanciadas por particulares numéricamente distintos.

La lógica de plurales deja incólumes las leyes básicas de la relación de identidad. Desde luego, si es verdad que las $x x$ son idénticas a las $y y$, entonces se satisface Reflexividad, $P I I$, y las demás consecuencias lógicas que se siguen de estas dos leyes básicas. La lógica de plurales está construida sobre la base de la lógica de singulares. Para que las $x x$ sean idénticas a las $y y$; para que $x$ sea una de las $y y$; para que expresiones plurales refieran a varios objetos; para que propiedades plurales sean instanciadas por varios objetos; etc., antes tiene que haber un objeto numéricamente idéntico a sí mismo y un segundo objeto idéntico sí mismo y distinto al primero. Pero no sucede lo mismo con el tipo híbrido de identidad propuesto por CIF, donde el valor de una de las variables es singular y los valores de las otras variables son plurales. Donde CIF falla de manera evidente es en la satisfacción de Simetría, pues si $x x \mathrm{C} y={ }_{\mathrm{df}} x x=y$, entonces debería seguirse que $y \mathrm{C} x x$. ¡Pero obviamente $y$ no compone las $x x$ ! ¿Cómo evitar esta absurda consecuencia? Respuesta de CIF: ... ¡evitándola! El mismo Bohn reconoce que "debemos restringir el sistema formal para evitar obtener $y C x x$ (que es un sinsentido mereológico)" (2014, 144n). Pero dado que ese "sinsentido mereológico" sí se sigue como consecuencia lógica, ¿cómo justificar la restricción del sistema formal? La restricción se hace necesaria solo para que la identidad entre composición e identidad sea viable. Estamos frente a un ajuste puramente ad-hoc. Literalmente, se trata de forzar los conceptos composición e identidad hasta hacerlos irreconocibles, de modo que entonces podamos sostener que son idénticos. CIF introduce una ambigüedad donde no lo hay y luego se ve forzada a excluir consecuencias lógicas que, en principio, se siguen perfectamente. Y ambos movimientos no parecen tener otra justificación que acomodar la idea de que identidad es una relación genérica y composición una especie de la misma. Lo único que se me ocurre para explicar estos movimientos-no para justificarlos-es apelar a un rasgo psicológico. Así como muchas veces la búsqueda de parsimonia ontológica o de hechos fundamentales no es más que el síntoma de cierta 
forma de ansiedad ontológica que suele aquejar al metafísico, creo que los esfuerzos por entender composición como identidad se deben también a una forma de ansiedad ontológica, a ciertas pulsiones parmenideanas que suelen habitar en todo metafísico: la pretensión de comprenderlo todo en términos de identidad. Pero claramente no todo puede ser comprendido en términos de identidad. Cierto, identidad es una relación básica: sin apelar a ella, nada admitiría ser pensado. Pero no todo puede ser reducido a ella. La relación de composición está llamada a hacer trabajo adicional que simplemente no puede ser hecho por la relación de identidad. Vale para composición lo mismo que otros han dicho con respecto a predicación o constitución material. Se trata de relaciones estrechamente conectadas con la relación de identidad, pero no se trata de las mismas relaciones.

En segundo lugar, CIF no parece hacer mucho por despejar la incoherencia inicial. Para efectos del argumento, aceptemos, como lo hace CIF, que cuando decimos que Platón y Sócrates son SoPa, el "son" utilizado es simplemente la forma plural del "es" de identidad. Si este fuese el caso, entonces, como bien argumenta Yi (1999a, 146-149), en cualquier interpretación correcta del predicado plural "es uno de", SoPa debe ser uno de Platón y Sócrates. Pero claramente no es el caso: SoPa no es ni Platón ni Sócrates. Luego, es algo distinto. Por tanto, CIF es falsa, y necesariamente falsa. Una cosa, necesariamente, es distinta a dos cosas: es una contradicción lógica sostener que una cosa es idéntica a dos cosas. Sucede, como ya he dicho, que una fusión y sus partes son discernibles: no comparten todas sus propiedades. Y si son discernibles, entonces, por PII, no son idénticos. De hecho, es por estas mismas razones que el propio Lewis pareciera inclinarse a ratos por la tesis débil, CID (Lewis 1991, 84, 87).

¿Qué puede hacer CIF para evitar esta conclusión? Adoptar una estrategia que relativice la cardinalidad de las cosas. Esto es, adoptar una estrategia que permita que "la misma porción de Realidad" sea una cosa bajo un concepto y varias cosas bajo otro concepto. Quienes suscriben CIF sostienen que identidad y cardinalidad no se predican de los mismos tipos de relata. Creen que es posible, frente a una "misma porción de Realidad", afirmar identidad en términos absolutos y cardinalidad en términos relativos. Mientras que un enunciado de identidad predicaría algo sobre uno o más objetos, un enunciado de cardinalidad afirmaría que el concepto F "tiene n instancias". De esta forma, "la misma porción de Realidad" podría tener cardinalidad 1 bajo el concepto $\mathrm{F}$, pero cardinalidad 2 bajo el concepto G. A esto apunta Bohn cuando, al definir informalmente CIF, introduce un giro fregeano: "un todo y sus partes colectivamente son la misma cosa bajo dos modos de presentación" (Bohn 2014, 143, énfasis agregado). Lo mismo sucede cuando, al intentar bloquear el argumento de Yi, Bohn le acusa de no aplicar correctamente PII, pues "lo que decimos con verdad de una cosa suele (aunque no siempre) depender de cómo la conceptualizamos" (Bohn 2014, 147). Y Bricker sigue a Bohn de cerca al describir su propia versión de CIF: "Hablar del todo y hablar de las partes que lo componen son simplemente dos formas diferentes de hablar de la 'misma porción de Realidad"' (Bricker 2016, 266, énfasis agregado). Las cosas no tendrían cardinalidad absoluta. La cardinalidad de "la 
misma porción de Realidad" dependería de los modos de presentarla o de hablar de ella. No es raro que traigan a Frege en apoyo de esta tesis. Frege rechazó la idea de que los números fuesen propiedades de objetos. Entendió que los números eran objetos auto-suficientes y que las aserciones de cardinalidad no predicaban nada de uno o más objetos, sino que decían algo sobre un concepto: que el concepto F "tiene $\mathrm{n}$ instancias":

Cuando frente al mismo fenómeno exterior puedo decir con igual verdad: 'esto es un grupo de árboles' y 'esto son cinco árboles', o bien 'aquí hay cuatro compañías' y 'aquí hay 500 hombres', en tal caso, no se modifica ni lo individual ni la totalidad, el agregado, sino solo mi denominación. Pero esto solo es síntoma de que he reemplazado un concepto por otro. Con ello se nos sugiere... que al asignar un número se afirma algo sobre un concepto. (Frege 1980, 59, énfasis agregado)

La familiaridad entre lo sostenido por CIF y Frege resulta evidente. Lo que Lewis y Bricker llaman una "misma porción de Realidad", o lo que Frege llama un "mismo fenómeno exterior", no tendría cardinalidad simpliciter, sino que, por ejemplo, tendría cardinalidad 4 en relación al concepto "compañías", pero 500 en relación al concepto "hombres".

¿Cómo se puede resistir esta relativización de la cardinalidad? ${ }^{1}$ Por análogas razones a las que uno puede resistir la relatividad de la identidad, tal como lo argumentaron hace unas décadas Alston y Bennett (1984). El punto central del argumento de Alston y Bennett es el siguiente: una vez asegurada la referencia de los términos que flanquean el signo de identidad en una aserción de identidad, no es necesario ni suficiente apelar a un concepto sortal F, común a los referentes, para que dicha aserción tenga valor de verdad determinado. Y lo dicho para las aserciones de identidad vale también para las aserciones de cardinalidad: una vez asegurada la referencia de los términos de una aserción de cardinalidad, no es necesario ni suficiente apelar a un sortal F, común a los referentes, para que dicha aserción tenga valor de verdad determinado. Aquí, por supuesto, F no es un sortal "dummy" como objeto, individuo, o cosa, sino uno que proporciona condiciones de identidad más exigentes o discriminatorias. Pues si cualquier referente admite ser pensado como un objeto, individuo, o cosa, entonces las visiones que relativizan la identidad o la cardinalidad a un sortal $\mathrm{F}$ no admiten ser distinguidas de la visión absoluta, que es la aquí defendida (Alston y Bennett 1984, 558).

Lo dicho recién es obvio si es que hay términos singulares (nombres propios, deícticos, etc.) que refieren sin mediar sentidos fregeanos. $\mathrm{Si}$ “ $x$ ", " $y$ ", $\mathrm{y}$ " $z$ " son términos singulares que refieren directamente a tres cosas distintas, $x, y, \mathrm{y} z$, respectivamente, entonces

\footnotetext{
${ }^{1}$ Más que relativizar los predicados numéricos a un concepto, Frege cambió el sujeto de la predicación numérica. Pero como ambos caminos conducen a similares resultados podemos decir que Frege relativizó la cardinalidad como Geach (1967) relativizó la identidad (Alston y Bennett 1984, 554-557; Perry 1970; 1978).
}

Revista de Humanidades de Valparaíso, 2020, No 1, 17-40 
las aserciones " $x, y, z$ son distintos" $\mathrm{y}$ " $x, y, z$ son tres" tienen valor de verdad determinado. No es necesario ni suficiente apelar a un sortal común F para determinarlo. No es necesario ni suficiente preguntar “¿distintos qué?” ni “¿cuántos $F s$ ?” para responder que hay tres objetos distintos. Lo mismo sucede si " $x$ ", " $y$ ", y " $z$ " son términos que refieren directamente a uno y el mismo objeto.

Ese es el escenario fácil. Pero digamos ahora que toda referencia es siempre indirecta. Digamos que " $x$ " solo puede referir al objeto que nombra, $x$, por la vía de un sentido fregeano. De ahí no se sigue que apelar a un concepto sortal F sea necesario o suficiente para que aserciones de identidad o de cardinalidad tengan valor de verdad determinado. Si solo puedo referir a $x$ por la vía del sentido la manzana roja más jugosa, a $y$ por la vía del sentido el único perro salchicha albino, y a $z$ por la vía del sentido el inventor del telégrafo, de ahí no se sigue que las aserciones " $x, y, z$, son distintos" $\mathrm{y}$ " $x, y, z$ son tres" requieran de un sortal F que sea "común" a los tres individuos para que ellas tengan valor de verdad determinado. De hecho, ni siquiera está garantizado que exista tal sortal común. Por ejemplo, no se me ocurre un sortal F (no "dummy") que sea "común" a la manzana roja más jugosa, el único perro salchicha albino, y el inventor del telégrafo; y si es que lo hay, seguramente no es difícil encontrar un ejemplo de individuos que se resistan a caer bajo un sortal F (no "dummy") que sea "común" a todos ellos. El punto es que si la referencia de nuestros términos singulares está asegurada, entonces las aserciones de identidad y cardinalidad tienen valor de verdad determinado. Obviamente, si $x=y=z$, entonces, por PII, todos los conceptos aplicables a $x$ serán conceptos aplicables a $y$ y a $z$, pero eso no significa que alguno de esos conceptos deba ser agregado a los enunciados de identidad o cardinalidad en cuestión para que estos tengan valor de verdad determinado. Cierto, a veces apelar a un concepto $\mathrm{F}$ ayuda a determinar la referencia de nombres propios como " $x$ ". Pero aun cuando ello fuese necesario para determinar la referencia de los términos singulares involucrados, no es algo que también sea necesario para determinar el valor de verdad de las aserciones de identidad o cardinalidad en que dichos términos figuran. Pues, una vez determinada la referencia de los términos que figuran en una aserción de identidad o de cardinalidad, no es algo indeterminado si acaso los términos refieren al mismo objeto o a distintos objetos, o si acaso refieren a uno o a varios objetos. Así, si tanto " $x$ " como " $y$ " refieren singularmente, sea directamente o indirectamente, es algo totalmente determinado si acaso $x=y$ o no, y si acaso $x$ e $y$ "son" uno o no. Si " $x$ " refiere al mismo objeto que " $y$ ", entonces refieren a uno y el mismo objeto; si " $x$ " no refiere al mismo objeto que " $y$ ", entonces refieren a dos objetos distintos. Estas reflexiones nos permiten detectar, de paso, el error en el que Frege incurrió al sostener lo siguiente:

Mientras que 'Solón era sabio’ y ‘Tales era sabio’ pueden reunirse en ‘Solón y Tales eran sabios', no puede decirse, en cambio, 'Solón y Tales eran uno'. No se podría comprender la imposibilidad de esto si 'uno' fuera, igual que 'sabio', una propiedad tanto de Solón como de Tales. (1980, 40-41) 
Su error fue el siguiente. Es claro que las oraciones "Solón era uno" y "Tales era uno" no "pueden reunirse" en "Solón y Tales eran uno". Si "Solón" refiere a un objeto distinto al que refiere "Tales", entonces la oración que "las reúne" es "Solón y Tales eran dos". "Eran dos" es un predicado que expresa una propiedad plural, y obviamente no puede ser dicho con verdad de un solo objeto. Pero sucede lo mismo con "eran sabios": se dice con verdad de más de un objeto. "Solón era sabio" y "Tales era sabio" no "se reúnen" en la oración "Solón y Tales era sabio", sino en la oración "Solón y Tales eran sabios".

Ahora bien, no solo no es necesario apelar a un sortal F para determinar el valor de verdad de una aserción de identidad o cardinalidad. Tampoco es suficiente. Pues incluso si se nos provee de un concepto F para contar objetos distintos, y dicho sortal es "común" a ellos, todavía puede resultar confuso determinar qué objetos se nos está pidiendo contar, como lo muestra este tradicional ejemplo:

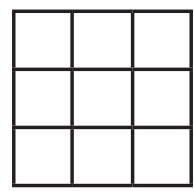

Si alguien apunta a la figura de arriba y pregunta “¿cuántos objetos distintos hay ahí?”, es claro que nos va a resultar difícil dar una respuesta. Pero nuestra situación no será mucho mejor si esa persona nos provee de un concepto sortal F "común" a los objetos a contar y pregunta "¿cuántas instancias distintas de cuadrado hay ahí?”. La cuestión no es clara: no porque no tengamos un término sortal F "común" a nuestro alcance, pues de hecho lo tenemos, sino porque es difícil determinar la referencia de nuestros términos incluso si se nos provee de tal término sortal. El punto central sigue incólume: una vez determinada la referencia de los términos singulares que figuran en aserciones de identidad o de cardinalidad, se cumplen las condiciones necesarias y suficientes para que esas aserciones tengan valor de verdad determinado. Aun cuando pueda ser necesario apelar a un concepto sortal $\mathrm{F}$ para determinar la referencia de tales términos, no es necesario ni suficiente apelar a un concepto sortal F "común" a dichos términos para que las aserciones de identidad o de cardinalidad en las que ellos figuran tengan valor de verdad determinado. Las aserciones de identidad no son fórmulas incompletas o cuya verdad sea relativa a un sortal "F"; y lo mismo vale para las aserciones de cardinalidad.

Los defensores de CIF tienen su corazón divido: por un lado, aborrecen la relativización de la identidad; por otro lado, no tienen mayor problema en relativizar la cardinalidad. Así, por ejemplo, Bohn no tiene mayor problema en afirmar que "ninguna cosa ordinaria tiene una particular cardinalidad con independencia a cómo es conceptualizada", al mismo tiempo que afirma que la "identidad misma por supuesto que no es relacional" $(2014,145)$ y que la "[i]dentidad relativa es peor que la muerte" $(2014,146)$. Pero la tensión reclama ser disuelta: si, como parece ser el caso, identidad y cardinalidad están necesariamente conectadas, entonces o ambas son absolutas o ambas son relativas. CIF 
tiene que elegir entre relativizar la identidad además de la cardinalidad, o dejar de relativizar la cardinalidad y entenderla tan absoluta como la identidad. Si hace lo primero, entonces debe abrazar un relativismo ontológico más radical que el que está dispuesta a abrazar y seguramente debe poner en entredicho PII, tal como lo hace CIE. Si hace lo segundo, entonces debe admitir su derrota, pues se queda sin recursos para evitar la conclusión lógica según la cual es contradictorio que una cosa sea (idéntica a) varias cosas. Aquí he intentado argumentar en favor de la posición absoluta. El número de las cosas no depende de nuestras formas de concebir o de hablar, sino de qué es idéntico a qué y de qué es distinto a qué. Y como identidad y diferencia son cuestiones que pertenecen a las cosas mismas, su número también.

\section{Conclusión}

He dado cuenta de dos versiones de la tesis según la cual composición es identidad, y de las críticas que se les pueden formular a ambas versiones. La versión débil de la tesis (CID)-según la cual composición es ontológicamente inocente en virtud de ser análoga a identidad-incurre en una petición de principio o en un non sequitur: en lo primero, si es que incluye la inocencia ontológica dentro de los aspectos de semejanza; en lo segundo, si es que no la incluye. Por su parte, la versión fuerte de la tesis (CIF)-según la cual composición es una especie de la relación genérica de identidad y tan ontológicamente inocente como cualquier especie de identidad-es incoherente, pues una cosa no puede ser idéntica a varias cosas. Esa incoherencia solo puede ser removida si se relativizan tanto identidad como cardinalidad, o si se aceptan identidad y cardinalidad en términos absolutos. Pero hacer lo primero equivale a abrazar una versión extraña de la tesis (CIE), según la cual, entre otras cosas, los idénticos son discernibles (contra PII); y hacer lo segundo equivale a aceptar la tesis según la cual composición no es identidad.

\section{Agradecimientos}

Este trabajo fue realizado en ejecución del proyecto Fondecyt-Iniciación № 11160724 (Conicyt, Chile) durante una estadía de investigación en la Facultad de Filosofía de la Universidad de Oxford, Reino Unido (mayo-junio, 2018).

\section{Referencias bibliográficas}

Alston, W. y J. Bennett (1984). Identity and cardinality: Geach and Frege. Philosophical Review, 93, 553-567. https://doi.org/10.2307/2184827

Armstrong, D. M. (1997). A World of States of Affairs. Cambridge: Cambridge University Press. https://doi.org/10.1017/CBO9780511583308

Revista de Humanidades de Valparaíso, 2020, No 1, 17-40 
Baxter, D. L. M. (1988). Many-one identity. Philosophical Papers, 17, 193-216. https://doi. org/10.1080/05568648809506300

Baxter, D. L. M. (2014). Identity, discernibility, and composition. En A. J. Cotnoir y D. L. M. Baxter (eds.), Composition as Identity, pp. 244-253. Oxford: Oxford University Press. https://doi.org/10.1093/acprof:oso/9780199669615.001.0001

Black, M. (1952). The identity of indiscernibles. Mind, 61, 153-164. https://doi.org/10.1093/ $\operatorname{mind} / \mathrm{LXI} .242 .153$

Bohn, E. (2014). Unrestricted composition as identity. En A. J. Cotnoir y D. L. M. Baxter (eds.), Composition as Identity, pp. 143-165. Oxford: Oxford University Press. https://doi. org/10.1093/acprof:oso/9780199669615.001.0001

Boolos, G. (1984). To be is to be a value of a variable (or to be some values of some variables). The Journal of Philosophy, 81, 430-449. https://doi.org/10.2307/2026308

Bricker, P. (2016). Composition as a kind of identity. Inquiry: An Interdisciplinary Journal of Philosophy, 59, 264-294. https://doi.org/10.1080/0020174X.2015.1040447

Della Rocca, M. (2012). Rationalism, idealism, monism, and beyond. En E. Förster y Y. Melamed (eds.), Spinoza and German Idealism, pp. 7-26. Cambridge: Cambridge University Press. https://doi.org/10.1017/CBO9781139135139.002

Fine, K. (1994). Essence and modality. Philosophical Perspectives, 8, 1-16. https://doi. org/10.2307/2214160

Fine, K. (2009). The question of ontology. En D. Chalmers, D. Manley, y R. Wasserman (eds.), Metametaphysics: New Essays on the Foundations of Ontology, pp. 157-177. Oxford: Clarendon Press.

Frege, G. (1960). Translations from the Philosophical Writings of Gottlob Frege, eds. P. Geach y M. Black. Oxford: Basil Blackwell.

Frege, G. (1980). The Foundations of Arithmetic. Austin. Oxford: Basil Blackwell.

Geach, P. (1967). Identity. The Review of Metaphysics, 21: 3-12. https://doi.org/10.2307/20124493

Koslicki, K. (2008). The Structure of Objects. Oxford: Oxford University Press. https://doi. org/10.1093/acprof:oso/9780199539895.001.0001

Lewis, D. (1986). On the Plurality of Worlds. Oxford: Basil Blackwell.

Lewis, D. (1991). Parts of Classes. Oxford: Basil Blackwell.

Mason, H. T. (ed.) (1967). The Leibniz-Arnauld Correspondence. Manchester: Manchester University Press.

McDaniel, K. (2008). Against composition as identity. Analysis, 68, 128-133. https://doi. org/10.1093/analys/68.2.128

McDaniel, K. (2013). Existence and number. Analytic Philosophy, 54, 209-228. http s://d oi . org/10.1111/phib.12013 
McGinn, C. (2000). Logical Properties. Oxford: Clarendon Press. https://doi. org/10.1093/0199241813.001.0001

Merricks, T. (1999). Composition as identity, mereological essentialism, and counterpart theory. Australasian Journal of Philosophy, 77, 192-195. https://doi. $\operatorname{org} / 10.1080 / 00048409912348931$

Perry, J. (1970). The same F. The Philosophical Review, 79, 181-200. https://doi. org/10.2307/2183947

Perry, J. (1978). Relative identity and relative number. The Canadian Journal of Philosophy, 7: 1-14. https://doi.org/10.1080/00455091.1978.10716204

Platón (1988). Diálogos V: Parménides, Teeteto, Sofista, Político. Madrid: Gredos.

Priest, G. (2011). Beyond the Limits of Thought. Oxford: Oxford University Press. https://doi. org/10.1093/acprof:oso/9780199254057.001.0001

Rosen, G. y C. Dorr (2002). Composition as a fiction. En R. Gale (ed.), The Blackwell Guide to Metaphysics,pp.151-174.Oxford:BasilBlackwell.https://doi.org/10.1002/9780470998984. ch8

Russell, B. (2010). Principles of Mathematics. London: Routledge.

Schaffer, J. (2007). From nihilism to monism. Australasian Journal of Philosophy, 85, 175-191. https://doi.org/10.1080/00048400701343150

Schaffer, J. (2010a). Monism: the priority of the whole. Philosophical Review, 119, 31-76. https:// doi.org/10.1215/00318108-2009-025

Schaffer, J. (2010b). The internal relatedness of all things. Mind, 119, 341-376. https://doi. org $/ 10.1093 / \mathrm{mind} / \mathrm{fzq} 033$

Sider, T. (2001). Four-Dimensionalism: An Ontology of Persistence and Time. Oxford: Clarendon Press. https://doi.org/10.1093/019924443X.001.0001

Sider, T. (2013). Against parthood. En K. Bennett y D. Zimmerman (eds.), Oxford Studies in Metaphysics 8, pp. 237-293. Oxford: Oxford University Press. https://doi.org/10.1093/ acprof:oso/9780199682904.003.0006

Sider, T. (2014). Consequences of collapse. En A. J. Cotnoir y D. L. M. Baxter (eds.), Composition as Identity, pp. 211-221. Oxford: Oxford University Press. https://doi.org/10.1093/ acprof:oso/9780199669615.001.0001

Simons, P. (1987). Parts: A Study in Ontology. Oxford: Clarendon Press.

Turner, J. (2014). Donald Baxter's composition as identity. En A. J. Cotnoir y D. L. M. Baxter (eds.), Composition as Identity, pp. 225-243. Oxford: Oxford University Press. https://doi. org/10.1093/acprof:oso/9780199669615.001.0001

van Inwagen, P. (1994). Composition as identity. Philosophical Perspectives, 8, 207-220. https:// doi.org/10.2307/2214171 
van Inwagen, P. (2002). The number of things. Philosophical Issues, 12, 176-196. https://doi. org/10.1111/j.1758-2237.2002.tb00066.x

Wallace, M. (2011a). Composition as identity: I. Philosophy Compass, 6, 804-816. https://doi. org/10.1111/j.1747-9991.2011.00431.x

Wallace, M. (2011b). Composition as identity: II. Philosophy Compass, 6, 817-827. https://doi. org/10.1111/j.1747-9991.2011.00430.x

Wiggins, D. (2016). Continuants: Their Activity, Their Being and Their Identity. Oxford: Oxford University Press. https://doi.org/10.1093/acprof:oso/9780198716624.001.000

Yi, B.-U. (1999a). Is mereology ontologically innocent? Philosophical Studies, 93, 141-160. https://doi.org/10.1023/A:1004274912342

Yi, B.-U. (1999b). Is two a property? The Journal of Philosophy, 96, 163-190. https://doi. org/10.2307/2564701

Yi, B.-U. (2005). The logic and meaning of plurals: part I. Journal of Philosophical Logic, 34: 459-506. https://doi.org/10.1007/s10992-005-0560-9

Yi, B.-U. (2006). The logic and meaning of plurals: part II. Journal of Philosophical Logic, 35, 239-288. https://doi.org/10.1007/s10992-005-9015-6

Yi, B.-U. (2014). Is there a plural object? En A. J. Cotnoir y D. L. M. Baxter (eds.), Composition as Identity, pp. 169-191. Oxford: Oxford University Press. https://doi.org/10.1093/ acprof:oso/9780199669615.003.0009 